\title{
MAGNETIC BEHAVIOR OF COMPACT AND CONVENTIONAL SINGLE TOWER TRANSMISSION LINE
}

\author{
A. A. Dahab \\ Department of Electrical Engineering, Faculty of Engineering \\ Minoufiya University, Shebin El-Kom, Egypt
}

\begin{abstract}
The paper investigates the magnetic flux density levels under and around a single tower transmission line. The single tower is a tower carrying transmission circuits with negligible mutual effect from any other circuits. Three cases for a single tower carrying a single circuit are considered. The first is a conventional delta which exhibits the highest level patterns. The second is a compact inverted delta which exhibits the optimal level patterns. The third is a compact delta which falls in between. A single tower carrying a conventional double 3-phase circuit is compared with a 6-phase converted circuit having the same configuration. These two cases are compared with a compact design 6-phase single tower transmission line. The 6-phase compact circuit exhibits the optimal performance. The conventional converted 6-phase circuit exhibits the highest density level patterns. The conventional double three phase circuit falls in between.

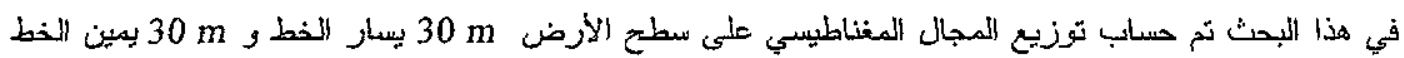

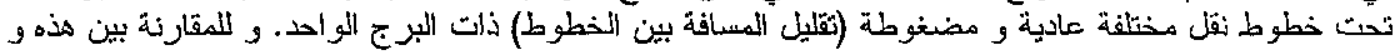

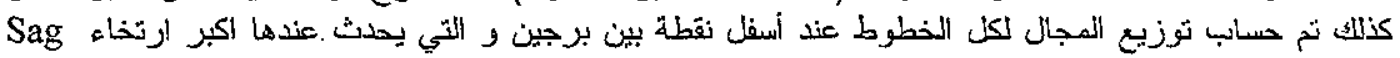
و النفس الميار .

و تم استخدام الخطوط التنالية بغرض المقارنة و أستخدام أفضلها:

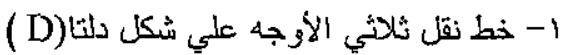

(CD) با

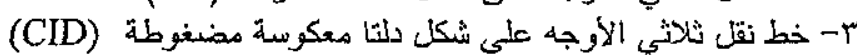

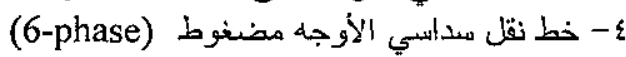

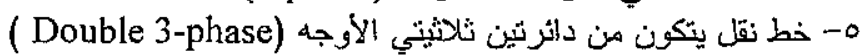

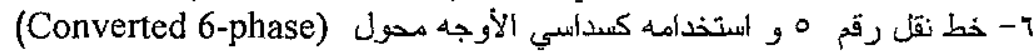

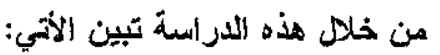

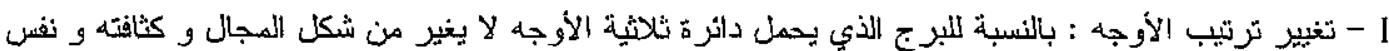

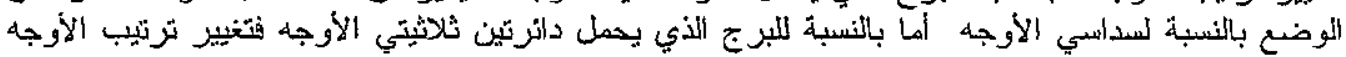

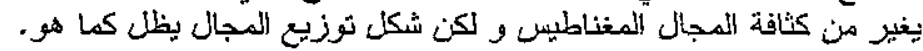

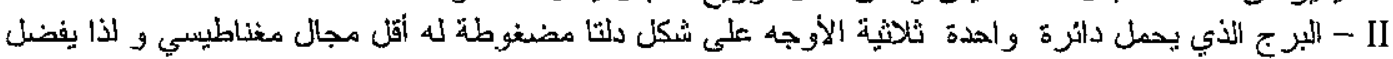

في المشاريع الجديدة.

- III

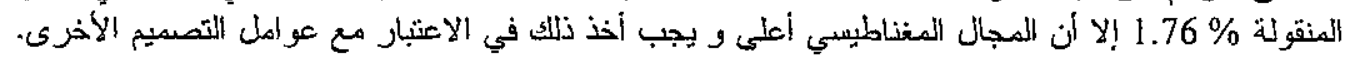

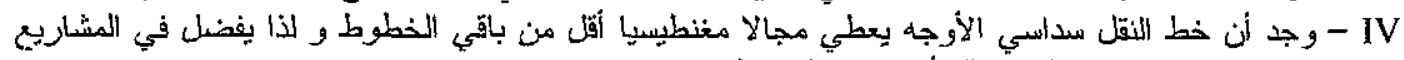
الجديدة كما في بعض البلدان مثل أمريكا و ألبرازيل.
\end{abstract}

Keywords: Compact transmission lines, Double three-phase circuit lines, Single towers, Six phase Circuits.

Engineering Research Journal, Vol.28, No. 4 October 2005, PP 329-334 


\section{INTRODUCTION}

There have been several debates about the biological effects of exposure to electric and magnetic fields (EMF) and their possible detrimental effects on human and animal health. However, after more than twenty years of research, it has not been conclusively demonstrated that any such detrimental effects exist. Statistical studies on children with leukemia have suggested that there is an association between the proximity of distribution lines to houses, where children live, and the disease. Attempts to correlate this directly with the magnetic field from the lines have not been successful. Further, it has not been possible so far to prove conclusively that magnetic fields can cause cancer or promote its growth [1-4].

Many studies have been carried out to evaluate the magnetic field distribution under different line configurations using various computational methods. These studies cover three-phase circuits and sixphase circuits. The magnetic field density under and around a single tower transmission line (i.e. $30 \mathrm{~m}$ left and $30 \mathrm{~m}$ right of the center line of the tower) is studied.

In this paper a single tower transmission line is a one with negligible mutual effect from any other circuits. This negligibility is achieved when the other transmission lines are $100 \mathrm{~m}$ more far from the center line of the tower under study [5]. The single tower carries a single 3-phase circuit or a double 3-phase circuit or a six-phase circuit. The magnetic field density under mid-span with minimum safe clearance to ground is considered in this paper. This is because it exhibits the highest density levels [5].

Three configurations of a single 3-phase $500 \mathrm{kV}$ circuit carrying a single tower are studied. The first is a conventional delta (D) configuration, Fig. 1a. The second is a compact delta (CD) configuration, Fig.lb. The third is a compact inverted delta (CID) configuration, Fig.1c. The phase conductors of the above mentioned three configurations consist of four bundled conductors $(4 \times 30 \mathrm{~mm}$ diameter $)$ per phase. Each phase bundle carries $1000 \mathrm{~A}$ [6]. A conventional

$115 \mathrm{kV}$ double 3-phase circuit (3D) configuration carried by single tower is also studied, Fig. 2a. This configuration is compared with a 6-phase circuit (6Con) carried by the above mentioned single tower, Fig $2 b$. This configuration is by conversion of the double 3-phase circuit using the same tower and the same conductors and the same current. This converted six-phase circuit is chosen normally to make advantage of the $76 \%$ increase in power handling capability without bothering the exiting accessible corridor [7].

The last two configurations are compared with a compact 6-phase circuit $(6 \mathrm{Com})$ carried by single tower, Fig. $2 \mathrm{c}$, having the same conductors and the same current as the two above mentioned cases. This is a favorable choice for new projects because it helps to reduce the problem of the right of way.

The phase conductors of the above mentioned three configurations consist of one conductor of $30 \mathrm{~mm}$ diameter per phase. Each phase conductor carries $1000 \mathrm{~A}$ [8-10].

The results in this paper help the designers to approach the optimal design for both existing and future projects.

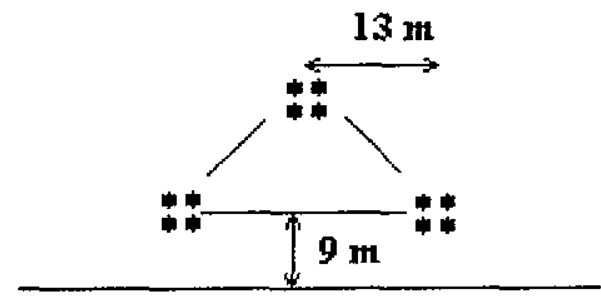

a

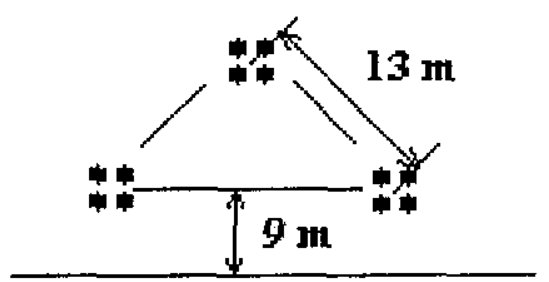

b

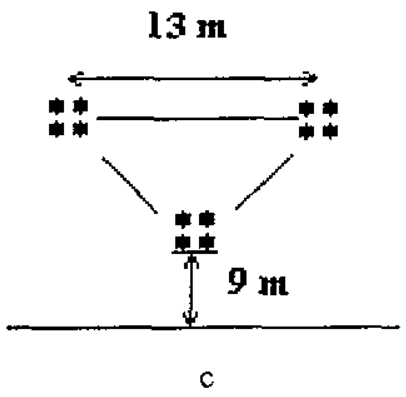

Fig 1: A single tower carrying a single circuit configuration:

a. Conventional delta (D) configuration

b. Compact delta (CD) configuration

c. Compact inverted delta (CID) configuration 


\section{METHOD OF CALCULATION}

The magnitude of the magnetic field intensity $d \vec{H}_{2}$ at a point 2 produced by a differential filamentary conductor $d \vec{L}_{1}$ carrying current $I_{1}$ is, Fig. $3,[11]$

$$
d \vec{H}_{2}=\frac{I_{1} d \vec{L}_{1} \times \hat{a}_{R_{12}}}{4 \pi R_{12}{ }^{2}}
$$

where;

$R_{12}$ : The normal distance from the differential element $d \vec{L}_{1}$ to the point 2 .

$\widehat{a}_{R_{\mathrm{t} 2}}:$ A unit vector normal to the differential element and the distance $R_{12}$.

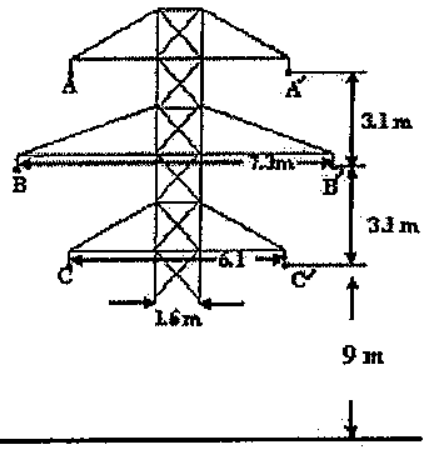

a : Conventional double 3-phase circuit (3D)

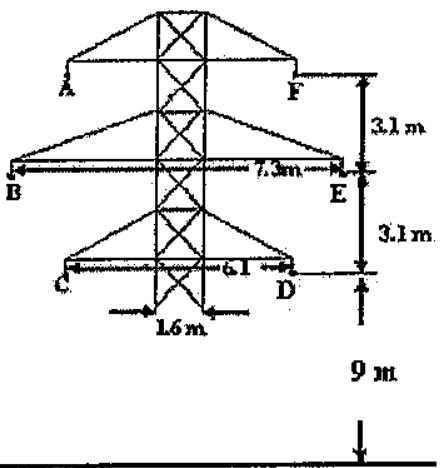

b : Conventional converted 6-phase circuit (6Con)

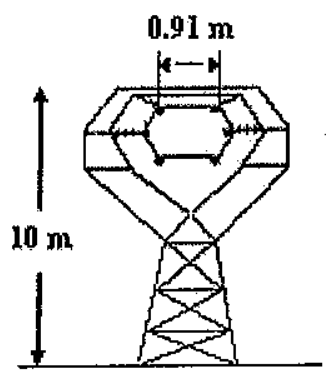

c : Compact 6-phase configuration (6Com)

Fig 2: A single tower carrying double 3-phase or 6phase
The transmission line current carrying conductor can be considered as an infinitely long straight filament carrying a root mean square current $I_{1}$, Fig. 4,

Equation (1) can be developed and the magnetic field intensity $\vec{H}_{p i}$ at point $\mathrm{P}$ is [11];

$$
\vec{H}_{p i}=\frac{I_{i}}{2 \pi R_{12}} \hat{a}_{\phi} \quad A / m
$$

where;

$\widehat{a}_{\phi}:$ A unit vector normal to the distance $R_{12}$

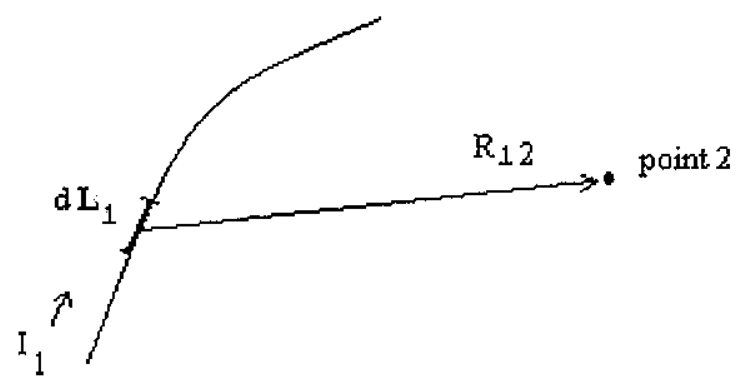

Fig 3: Illustration of Equation (1)

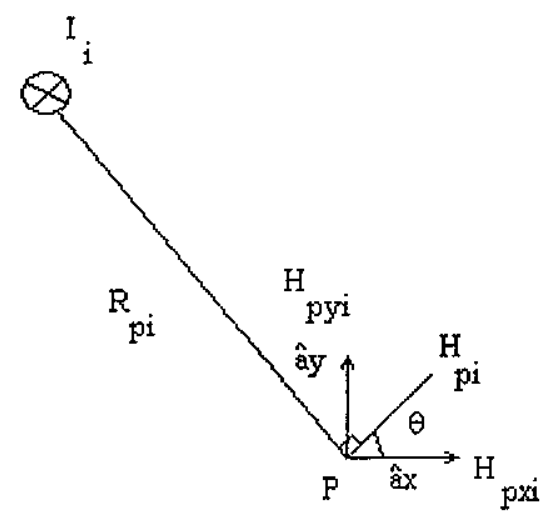

Fig 4: Illustration of Equations (2)

The three-phase circuits consist of three currentcarrying conductors with $120^{\circ}$ phase shift between the currents. The 6- phase circuits consist of six current-carrying conductors with $60^{\circ}$ phase shift between the currents. The resultant magnetic field intensity at any point, Say $P$, is the vectorial summation of the magnetic field intensity caused by $\mathrm{N}$ current-carrying conductors of the single tower. Horizontal and vertical components, Equation.2, of the magnetic field intensity at point $\mathrm{P}$ as a result of a current, say $\mathrm{I}_{\mathrm{i}}$, are; 


$$
\begin{aligned}
& H_{x p i}=\frac{I_{i} \cos \theta}{2 \pi R} \hat{a}_{x i} \\
& H_{y p i}=\frac{I_{i} \sin \theta}{2 \pi R_{p i}} \hat{a}_{y}
\end{aligned}
$$

The resultant magnetic field intensity of $\mathrm{N}$ currents carried by $\mathrm{N}$ conductors of a single tower is;

$$
H_{t p}=\sqrt{\left(\sum_{i=1}^{N} H_{x p i}\right)^{2}+\left(\sum_{i=1}^{N} H_{y p i}\right)^{2}}
$$

The resultant magnetic field density at point $P$ is given by:

$$
\begin{aligned}
& B_{t p}=\mu_{o} H_{t p} \quad T \\
& \mu_{o}=4 \pi \times 10^{-7} \quad \mathrm{H} / \mathrm{m}
\end{aligned}
$$

\section{A SINGLE CIRCUIT CARRYING A SINGLE TOWER CONFIGURATION}

In Fig. 5, the solid curve represents the D configuration, the stars curve represents the $C D$ configuration, the circles curve represents the CID configuration. Comparing these curves shows that the $D$ configuration exhibits the highest density levels at all locations. It shows also that CID configuration exhibits the lowest density levels at all locations.

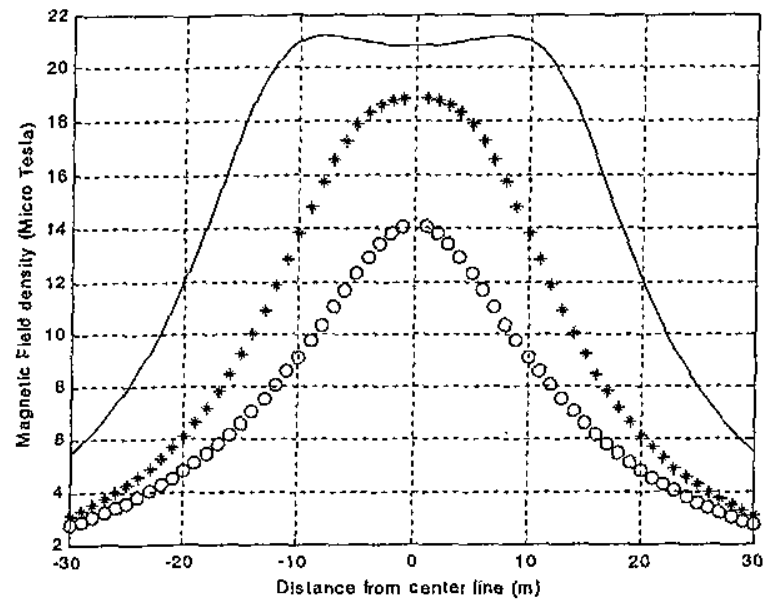

Fig 5: Magnetic flux density distribution of: $\mathrm{D}(-), \mathrm{CD}(*), \mathrm{CID}(\mathrm{o})$

The $D$ curve shows a spread peak around the center line of $21.5 \mu T$ average value. Ten meters far from the center line, the density starts to drop sharply down to about $5.5 \mu T$ at $30 \mathrm{~m}$ distance from the center line.

Both the $C D$ and the CID curves have a single peak exactly at the center line. The curve around the peak in the CID is sharper than that of the CD. The CD peak value amounts to $19 \mu T$ and the lowest value drops down to $3 \mu T$ at $30 \mathrm{~m}$ far from the center line. The CID peak value amounts to $14.25 \mu T$ and the lowest value drops to $2.5 \mu \mathrm{T}$ at $30 \mathrm{~m}$ far from the center line.

The CD configuration reduces the peak by $10.6 \%$ and the CID configuration reduces the peak by about $34 \%$ and the lowest level, at $30 \mathrm{~m}$ far from the center line, reduces by more than $50 \%$ all compared with the D configuration.

It is important to observe that CID configuration has one phase nearer to the ground while the other two phases are at higher levels. The CD configuration has two phases nearer to the ground level and one phase at higher level.

The change in phase arrangement as transposition takes place is studied and no change is noticed in magnetic field density levels and subsequently the curves are not needed for this case.

\section{SINGLE TOWER CARRYING DOUBLE THREE PHASE CIRCUITS OR A SIX- PHASE CIRCUIT}

Figure 6 shows the distribution of the magnetic field density under $3 \mathrm{D}$ configuration. The solid curve is for $A B C-A B C$ phase arrangement of the two three phase circuits top to bottom. The star curve is for $A B C$ CBA phase arrangement of the two three phase circuits top to bottom. Comparing the two curves shows that changing the phase arrangement leads to a change in the magnetic field density distribution, in this case $A B C-C B A$ arrangement in comparison with the $A B C-A B C$ arrangement exhibits a $5 \mu T$ reduction in the peak value and $1.7 \mu T$ reduction in the minimum value.

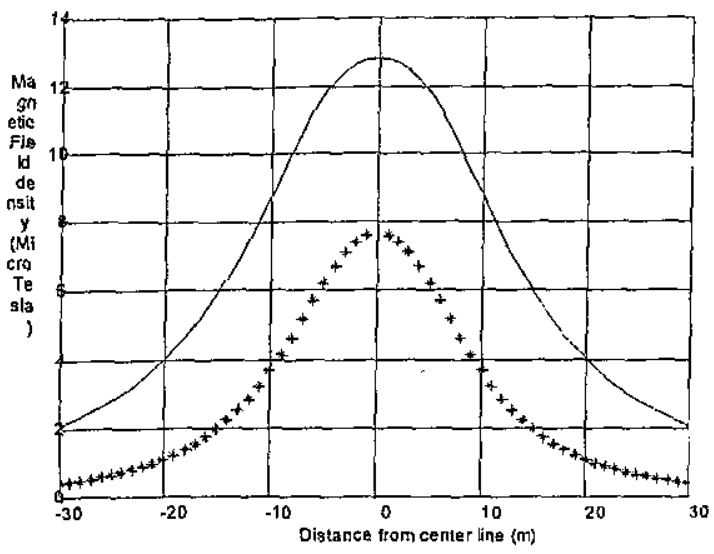

Fig 6: Magnetic flux density level under 3D circuit $A B C-A B C$ phase arrangement (-) $\mathrm{ABC}-\mathrm{CBA}\left({ }^{*}\right)$ 


\section{A. A. Dahab, "Magnetic Behavior Of Compact and Conventional Single Tower Transmission Line"}

The phase arrangement changes as transposition takes place. Subsequently, worst case should be considered to make sure that its level is below the maximum permissible limits.

Changing the phase arrangement in the six phase circuits is tested and it does not result in any change in the magnetic field density patterns. Subsequently, no curves are needed.

Figure 7 shows three magnetic field density distribution curves. The solid curve is for 3D configuration with $\mathrm{ABC}-\mathrm{ABC}$ phase arrangement taken as a reference. The star curve is for $6 \mathrm{Con}$ configuration. The circles curve is for $6 \mathrm{Com}$ configuration.

The 6 Con configuration exhibits the highest density level at all points. A single peak of $20 \mu T$ occurs exactly at the center line. The density falls sharply down to $2.5 \mu T$ at $30 \mathrm{~m}$ far from the center line.

The 6 Com configuration exhibits the lowest density level curve at all points. The 6 Com curve has a single peak of $5.9 \mu T$ exactly at the center line. The magnetic field density falls sharply up to $15 \mathrm{~m}$ far from the center line and then slowly down to $0.4 \mu T$ at $30 \mathrm{~m}$ far from the center line. Comparing the $6 \mathrm{Com}$ with the reference case, the peak drops by $55 \%$ and the minimum drops by $80 \%$. Comparing the 6 Con with reference case the peak increases by about $54 \%$ and the minimum increases by about $20 \%$.

The 3D configuration falls in between with a single peak of $12.8 \mu T$ falling exactly at the center line. The minimum is $2.1 \mu T$ at $30 \mathrm{~m}$ far from the center line.

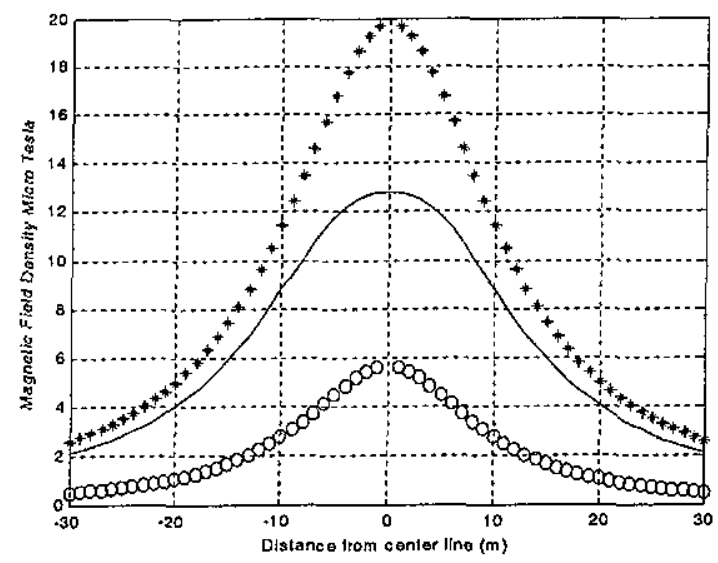

Fig 7: $3 \mathrm{D}$ circuit with $\mathrm{ABC}-\mathrm{ABC}$ phase arrangement $(-), 6$ Con circuit $(*), 6$ Com circuit (o)

\section{CONCLUSION}

I. The Single circuit carrying a single tower configuration

- The change in phase arrangement does not affect the shape or the level of the magnetic field density patterns.

- The compact inverted delta is highly recommended for new projects because it exhibits the optimal magnetic field density levels at all locations and minimize the problems of the right of way.

II. The double three-phase circuit configurations and the six-phase circuit configurations both carrying a single tower configuration.

- The phase arrangement in the double three phase circuits give similar patterns but with different leveis. Careful examination of different flux density level patterns should be studied as phase arrangement changes when transposition takes place. This is necessary to insure that worst case pattern levels are lower than maximum permissible levels.

- For the six phase circuit, the change in phase arrangement does not affect the shape or the level of the magnetic flux density patterns.

- Converting the existing double three phase circuits to a six phase circuit is recommended to increase power handling capacity by $76 \%$, but it also increases the magnetic flux density levels. Subsequently, careful examination of magnetic flux density levels of the converted circuit is necessary to insure that it is below the maximum permissible levels.

- The compact six phase circuit is highly recommended for new projects because it exhibits the optimal magnetic field density patterns, It can handle higher power and reduces the problem of the right of way.

\section{REFERENCES}

[1] W. Dab, J. Lambrozo, and M.Souques, "Magnetic fields and cancer: Recent studies in adults", Cigre, panel 1-03, Paris, 1994.

[2] G. O. Young, "Synthetic structure of industrial plastics (Book style with paper title and editor)," in Plastics, 2nd ed. vol. 3, J. Peters, Ed. New York: McGraw-Hill, 1964, pp. 15-64.

[3] T. W. Dawson, K. Caputa, and M. Stuchly, "Influence of Human Model Resolution on Computed Currents Induced in Organs by $60-\mathrm{Hz}$ 
Magnetic Fields", Bioelectromagnetics, Vol. 18, PP. 478-490, 1997.

[4] C. I. Durkin, R. P. Fogarty, T. M. Halleran, and D. A. Mark, "Five Years of Magnetic Field Management", IEEE Power Delivery, Vol. 10, No. 1, PP. 219-228, Jan. 1995.

[5] A. S. Farag, et al., "Magnetic Field Measurement and Management in and Around Substations in Saudi Arabia", ClGRE, Paper 36201, Paris, 1998.

[6] H.Saddat, "Power System Analysis", McGraw Hill Book Company, 1999.

[7] M. A. Abd-Allah and A. S. Alghamdi, "Magnetic Field Mitigation Using Line Compaction", The $8^{\text {th }}$ International Middle-East Power Systems Conference (MEPCON 2001), Cairo, Egypt, Dec. 2001.
[8] J. R. Sterwart, J. Oppel, G. C. Thomann, T. F Dorazio, M. T. Brown," Insulation Coordination , Environmental and System Analysis of Existing Double Circuit Line Reconfigured to Six-Phase Operation", IEEE Transactions on Power Delivery, Vol. 7, No. 3, PP. 1628-1633, July 1992.

[9] M. O: Melo, et al., "Electric and Magnetic Fields of Compact Lines", IEEE Trans. On Power Delivery, Vo. 14, No. 1, PP. 200-204, Jan. 1999.

[10] F.Amoura, A.M.Basha, Said E.Emasry, Anis Afzal, " High Phase Order Transmission (6phase)- An overview", Water and Energy International Journal, Vol.57; No.1,JanuaryMarch 2000, ISSN 0972-057X.

[11] W. Hayt, jr, "Engineering Electromagnetics", McGraw-Hill Book Company, 1989. 